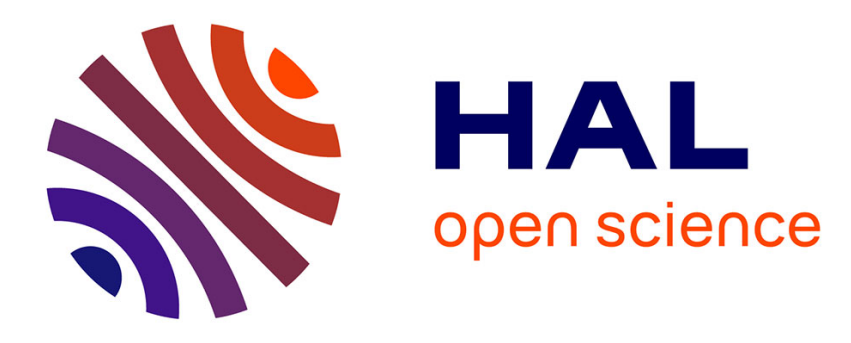

\title{
Arc Consistency Projection: A New Generalization Relation for Graphs
}

Michel Liquière

\section{To cite this version:}

Michel Liquière. Arc Consistency Projection: A New Generalization Relation for Graphs. ICCS: International Conference on Conceptual Structures, Jul 2007, Sheffield, United Kingdom. pp.333-346, 10.1007/978-3-540-73681-3_25. lirmm-00196399

\section{HAL Id: lirmm-00196399 https://hal-lirmm.ccsd.cnrs.fr/lirmm-00196399}

Submitted on 16 Sep 2019

HAL is a multi-disciplinary open access archive for the deposit and dissemination of scientific research documents, whether they are published or not. The documents may come from teaching and research institutions in France or abroad, or from public or private research centers.
L'archive ouverte pluridisciplinaire HAL, est destinée au dépôt et à la diffusion de documents scientifiques de niveau recherche, publiés ou non, émanant des établissements d'enseignement et de recherche français ou étrangers, des laboratoires publics ou privés. 


\title{
Arc Consistency Projection: A New Generalization Relation for Graphs
}

\author{
Michel Liquiere \\ LIRMM, \\ 161 Rue ada, \\ 34392 Montpellier cedex 5, \\ France \\ Liquiere@lirmm.fr
}

\begin{abstract}
The projection problem (conceptual graph projection, homomorphism, injective morphism, $\theta$-subsumption, OI-subsumption) is crucial to the efficiency of relational learning systems. How to manage this complexity has motivated numerous studies on learning biases, restricting the size and/or the number of hypotheses explored. The approach suggested in this paper advocates a projection operator based on the classical arc consistency algorithm used in constraint satisfaction problems. This projection method has the required properties : polynomiality, local validation, parallelization, structural interpretation. Using the arc consistency projection, we found a generalization operator between labeled graphs. Such an operator gives the structure of the classification space which is a concept lattice.
\end{abstract}

\section{Introduction}

The complexity of the computation of the generality relation between two relational descriptions, is a crucial problem. For conceptual graphs [1] this operation is named projection. Such an operation is linked to a classical problem in the graph community: the search for an homomorphism between two graphs. As stated in [2], "the elementary reasoning operation, projection is a kind of graph homomorphism that preserves the partial order defined on labels". The search for an homomorphism between a tree and a graph is polynomial but between general graphs, the problem is NP complete [3]. In conceptual graph community, different algorithms are proposed for the projection problem [456].

From another point of view, Inductive Logic Programming systems (ILP) commonly used a generality relation, a decidable restriction of logical implication named $\theta$-subsumption. The homomorphism is also directly linked to the $\theta$-subsumption operation [7]. In machine learning, the complexity of this operation has motivated the use of learning biases: syntactic biases (trees [8, specific graph 910]), efficient implementation [7] and approximation of $\theta$-subsumption [1].

Finally, the homomorphism is also linked to the classical constraint programming resolution (CSP) 12. This final link gives an interesting algorithmic point 
of view since CSP community has many results which improve the resolution algorithm.

In this paper, we propose to use a part of these three domains for a classical unsupervised machine learning problem. We represent each example by a labeled graph. We use a new generality relation named AC-projection based on the arc consistency algorithm [12] and we prove that the search space, for a relational machine learning classification problem, is a concept lattice [13].

\section{A New Projection: AC-Projection}

Any constraint satisfaction problem can be viewed as a "network" of variables and constraints. In this network each variable is connected to the constraints that involve it and each constraint is connected to the variables it involves. Among backtracking based algorithms for constraint satisfaction problems, algorithm employing constraint propagation, like forward checking and arc consistency [12, have had the most practical impact. These algorithms use constraint propagation (arc consistency) during a search to prune inconsistent values from the domains of the uninstantiated variables.

\subsection{AC-Projection and Arc Consistency}

In this paragraph we present the arc consistency using a graph notation. For other presentations see the books [3]12].

Notation. For a labelled directed graph, named digraph in this paper, G, we note $V(G)$ the set of vertices of $\mathrm{G}, A(G)$ the set of arcs of $\mathrm{G}, L(G)$ the set of labels of G. For a vertex $x \in V(G)$ we note $l(x) \in L(G)$ the label of x, $N(x)$ the set of all the neighbors of $\mathrm{x}, P(x) \subseteq N(x)$ the predecessors of $\mathrm{x}$ and $S(x) \subseteq$ $N(x)$ the successors of $\mathrm{x}$.

For a finite set $\mathrm{S}$ we note $2^{S}$ the set of all subsets of $\mathrm{S}$ (power set). In this paragraph we study some important properties of the arc consistency.

Definition 1 (labeling). Let $G_{1}$ and $G_{2}$ be two digraphs. We named labeling from $G_{1}$ into $G_{2}$ a mapping $\mathcal{I}: V\left(G_{1}\right) \rightarrow 2^{V\left(G_{2}\right)} \mid \forall x \in V\left(G_{1}\right), \forall y \in \mathcal{I}(x)$, $l(x)=l(y)$.

Thus for a vertex $x \in \mathrm{V}\left(G_{1}\right), \mathcal{I}(\mathrm{x})$ is a set of vertices of $G_{2}$ with the same label $l(x)$. We can think of $\mathcal{I}(x)$ as the set of "possible images" of the vertex $x$ in $G_{2}$. This first labeling is trivial but can be refined using the neighborhood relations between vertices.

Definition $2(\sim \succ)$. Let $G$ be a digraph, $V_{1} \subseteq V(G), V_{2} \subseteq V(G)$.

We note $V_{1} \sim \succ V_{2}$ iff

1) $\forall x_{k} \in V_{1} \exists y_{p} \in V_{2} \mid\left(x_{k}, y_{p}\right) \in A(G)$

2) $\forall y_{q} \in V_{2} \exists x_{m} \in V_{1} \mid\left(x_{m}, y_{q}\right) \in A(G)$.

In this definition we give a direct relation between two sets of vertices $V_{1}$ and $V_{2}$. So for each vertex $x_{k}$ of $V_{1}$ there is at least one vertex $y_{p}$ of $V_{2}$ which is a 
neighbor of $x_{k}:\left(\left(x_{k}, y_{p}\right) \in \mathrm{A}(G)\right)$ and all vertices of $V_{2}$ are a neighbor of, at least, one vertex of $V_{1}$ (oriented condition). This is not a one to one relation like the subgraph isomorphism.

Definition 3 (Consistency for one arc). Let $G_{1}$ and $G_{2}$ be two digraphs. We say that a labeling $\mathcal{I}: V\left(G_{1}\right) \rightarrow 2^{V\left(G_{2}\right)}$ is consistent with an arc $(x, y) \in A\left(G_{1}\right)$, iff $\mathcal{I}(x) \sim \succ \mathcal{I}(y)$.

In the example of Figure 1, a vertex is designated by a letter and a number: the letter is the label of the vertex and the number is only an identification number. In this example the labeling $\mathcal{I}: \mathcal{I}\left(a_{0}\right)=\left\{a_{4}, a_{10}\right\}$ and $\mathcal{I}\left(b_{1}\right)=\left\{b_{5}, b_{9}\right\}$, is consistent with the edge $\left(a_{0}, b_{1}\right)$ since $\mathcal{I}\left(a_{0}\right) \sim \mathcal{I}\left(b_{1}\right)$.

Definition 4 (AC-projection $\rightarrow$ ). Let $G_{1}$ and $G_{2}$ be two digraphs. A labeling $\mathcal{I}$ from $G_{1}$ into $G_{2}$ is an $A C$-projection iff $\mathcal{I}$ is consistent with all the arcs $e \in$ $A\left(G_{1}\right)$. We note it $G_{1} \rightarrow G_{2}$

The name "AC-projection" comes from the classical AC (arc consistency) used in 12$]$.

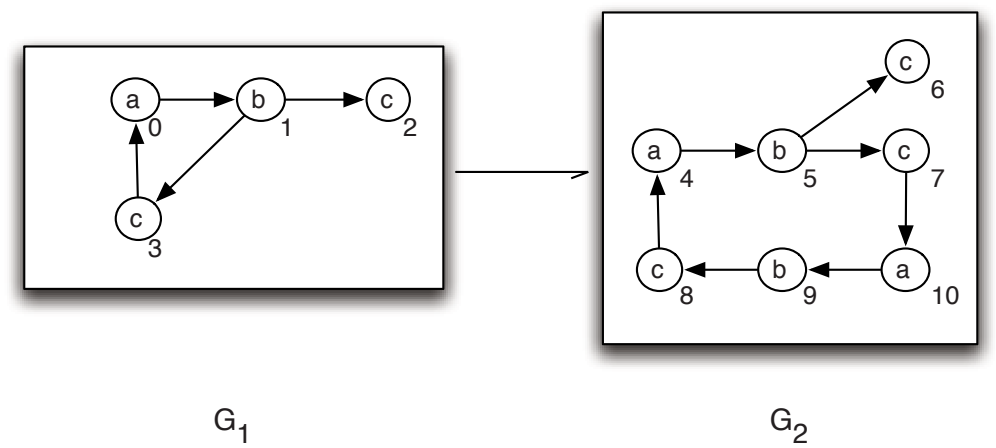

Fig. 1. AC-Projection: Example

Consider the labelling $\mathcal{I}\left(a_{0}\right):\left\{a_{4}, a_{10}\right\}, \mathcal{I}\left(b_{1}\right):\left\{b_{5}, b_{9}\right\}, \mathcal{I}\left(c_{2}\right):\left\{c_{6}, c_{7}, c_{8}\right\}, \mathcal{I}\left(c_{3}\right)$ : $\left\{c_{7}, c_{8}\right\}$. We verify $\mathcal{I}\left(a_{0}\right) \sim \succ \mathcal{I}\left(b_{1}\right), \mathcal{I}\left(b_{1}\right) \sim \succ \mathcal{I}\left(c_{2}\right), \mathcal{I}\left(b_{1}\right) \sim \succ \mathcal{I}\left(c_{3}\right), \mathcal{I}\left(c_{3}\right) \sim \succ \mathcal{I}\left(a_{0}\right)$. Then $\mathcal{I}$ is an AC-projection from $G_{1}$ into $G_{2}$ since $\mathcal{I}$ is a labelling consistent with all arcs of $G_{1}$.

\section{$2.2 \quad$ AC-Projection Properties}

We have defined a new mapping relation between graphs. In this paragraph we study the properties of this relation (complexity, interpretation).

We recall the classical homomorphism definition for digraphs. 
Definition 5 (homomorphism $\mapsto$ ). A homomorphism of a digraph $G_{1}$ to a digraph $G_{2}$ is a mapping of the vertex sets $f: V\left(G_{1}\right) \rightarrow V\left(G_{2}\right)$ which preserves the arcs and labels, i.e such that $(x, y) \in A\left(G_{1}\right) \Rightarrow(f(x), f(y)) \in A\left(G_{2}\right)$ and $\forall x$, $l(x)=l(f(x))$.

Notation: $G_{1} \mapsto G_{2}$

Note that for digraph $(\mathrm{f}(\mathrm{x}), \mathrm{f}(\mathrm{y})) \in \mathrm{A}\left(G_{2}\right)$ implies that $\mathrm{f}(\mathrm{x}) \neq \mathrm{f}(\mathrm{y})$, since each edge of $\mathrm{A}\left(G_{2}\right)$ consists of two distinct elements.

We have the following proposition which links the AC-projection to the Homomorphism.

Proposition 1. For two digraphs $G_{1}$ and $G_{2}$, if $G_{1} \mapsto G_{2}$ then $G_{1} \rightarrow G_{2}$.

Proof. See 3 ]

This proposition is the foundation of many CSP resolution methods. These methods are based on the classical arc consistency algorithm AC1 used in CSP, which has been improved ( $\mathrm{AC} 2 \ldots$ AC5), the actual minimal complexity is: $\mathrm{O}\left(e d^{2}\right)$ where $e$ is the number of arcs and $d$ the size of the largest domain 12 .

In our case, the size of the largest domain is the size of the largest subset of nodes with the same label. So an AC-projection between two digraphs can be computed in polynomial time.

\subsection{AC-Projection Algorithm}

We give a simple AC-projection algorithm for digraphs (based on AC1 algorithm [12]).

This algorithm Arc-Consistency takes two digraphs $G_{1}, G_{2}$ and tests if there is an AC-projection from $G_{1}$ into $G_{2}$. It begins by the creation of a first rough labeling $\mathcal{I}$ and reduces, for each vertex $\mathrm{x}$, the given lists $\mathcal{I}(\mathrm{x})$ to consistent lists using the procedure ReviseArc.

The consistency check fails if some $\mathcal{I}(\mathrm{x})$ becomes empty; otherwise the consistency check succeeds and the algorithm gives the labeling $\mathcal{I}$ which is an ACprojection $G_{1} \neg G_{2}$.

Procedure: ReviseArc

Data: An $\operatorname{arc}(\mathrm{x}, \mathrm{y}) \in \mathrm{V}\left(G_{1}\right)$

Data: A labeling $\mathcal{I}$ from $G_{1}$ into $G_{2}$

Data: A digraph $G_{2}$

Result: A new labeling $\mathcal{I}^{\prime}$ from $G_{1}$ into $G_{2}$

$\mathcal{I}^{\prime}:=\mathcal{I}$

$\mathcal{I}^{\prime}(\mathrm{x}):=\mathcal{I}(\mathrm{x})-\left\{\mathrm{x}^{\prime} \in \mathrm{V}\left(G_{2}\right) \mid \exists \mathrm{y}^{\prime} \in \mathcal{I}(\mathrm{y})\right.$ with $\left.\left(\mathrm{x}^{\prime}, \mathrm{y}^{\prime}\right) \in \mathrm{A}\left(G_{2}\right)\right\} ;$

$\mathcal{I}^{\prime}(\mathrm{y}):=\mathcal{I}(\mathrm{y})-\left\{\mathrm{y}^{\prime} \in \mathrm{V}\left(G_{2}\right) \mid \exists \exists \mathrm{x}^{\prime} \in \mathcal{I}(\mathrm{x})\right.$ with $\left.\left(\mathrm{x}^{\prime}, \mathrm{y}^{\prime}\right) \in \mathrm{A}\left(G_{2}\right)\right\} ;$

return $\mathcal{I}^{\prime}$ 


\section{Procedure: Arc-Consistency}

Data: Two digraphs $G_{1}$ and $G_{2}$

Result: An AC-projection $\mathcal{I}$ from $G_{1}$ into $G_{2}$ if there is one else an empty set $\emptyset$

// Initialisation

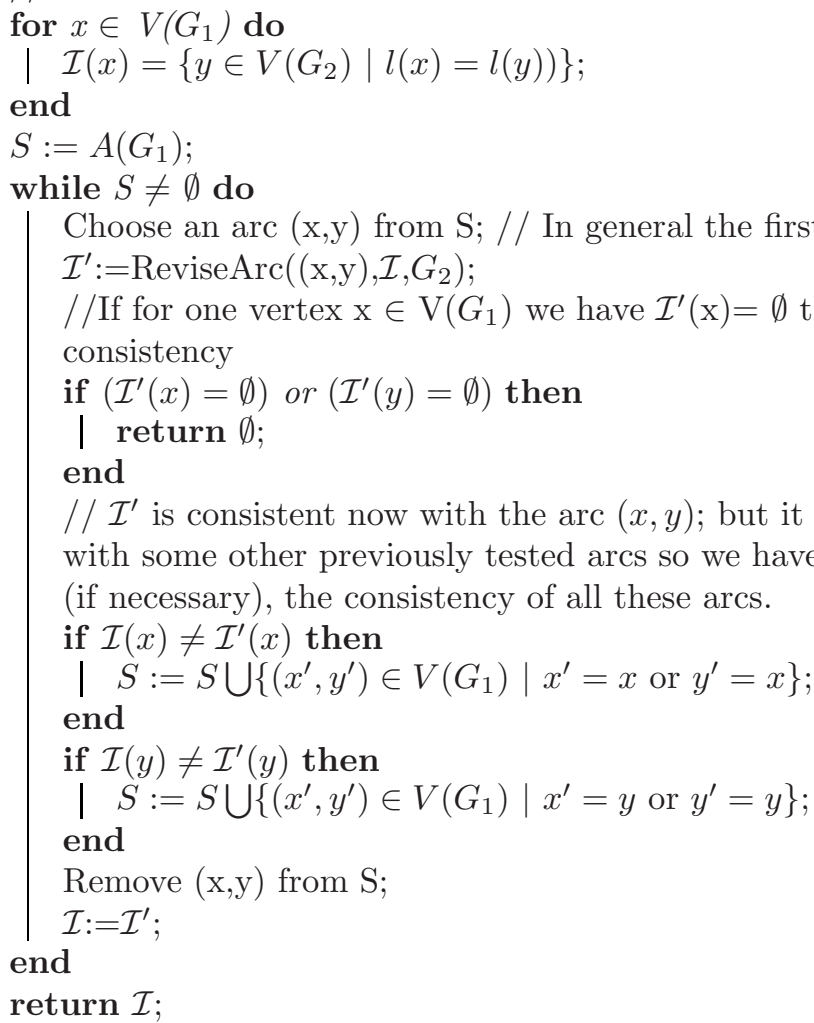

The Arc-Consistency algorithm has a polynomial time complexity 312 and gives, if there is one, an AC-projection $\mathcal{I}$ from $G_{1}$ into $G_{2}$ verifying: for all AC-projection $\mathcal{I}^{\prime}$ from $G_{1}$ into $G_{2}$, we have $\forall \mathrm{x} \in \mathrm{V}\left(G_{1}\right), \mathcal{I}^{\prime}(\mathrm{x}) \subseteq \mathcal{I}(\mathrm{x})[3]$.

\section{AC-Projection and Machine Learning}

In [10, we have studied the construction of a concept lattice, where the extension part is a subset of the set of example but where the intension part is described by a digraph. In the context of machine learning, the automatic bottom up construction of such a hierarchy can be viewed as an unsupervised conceptual classification method. In this paper the generalization partial order was based on homomorphism relation between digraph. To deal with the homomorphism complexity, we proposed a class of digraph with a polynomial homomorphism operation. This limit the generality of the description language. 
In that paper we propose to put the bias on the projection operator. Since the complexity of the AC-projection is polynomial, our idea is to use the ACprojection algorithm instead of the homomorphism projection.In doing so, we need a structural interpretation of the results. In the case of the subgraph isomorphism relation between two graphs, there is no interpretation problem, because it is an "inclusion" relationship. For the homomorphism relation the interpretation is less natural since two vertices can get the same image. The structural interpretation of the AC-projection seems unnatural. For example, see Figure 1 and seek for the substructures which are in $G_{1}$ and $G_{2}$.

In fact, in the paper [3], the author gives the following proposition:

Proposition 2. Let $G_{1}$ and $G_{2}$ be any labelled digraphs with $G_{1} \rightarrow G_{2}$. If an directed labeled tree $T$ satisfies $T \mapsto G_{1}$ then $T \mapsto G_{2} \cdot($ recall $\mapsto$ is the homomorphism relation)

A limited interpretation of the proposition 2 is: every subtree of $G_{1}$ has an homomorphic image in $G_{2}$. So all the covering trees of the digraph $G_{1}$ of the Figure 1. are homomorphic with $G_{2}$.

\subsection{AC-Projection and Generalization}

The basic building blocks of concept learning is the notion of example and description language. In our framework, each example, of the set of example, is described by one digraph. So we have a set of digraphs $\mathcal{E}$. We want to find a set of labeled digraphs which have an AC-projection with a subset of the labelled

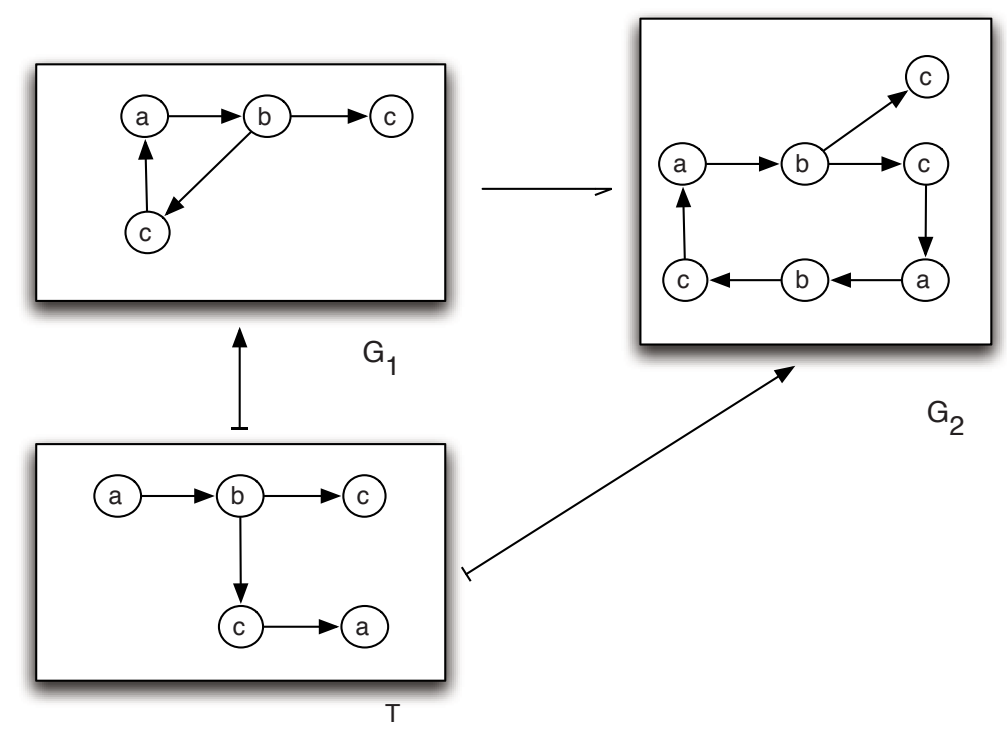

Fig. 2. AC-projection and interpretation 
digraphs in $\mathcal{E}$. It is a classical unsupervised classification problem. A generalization algorithm uses a generalization operator: from two graphs we search for the more specific graph which generalizes two graphs (least general generalization [14]). Our generalization order will use the AC-projection relation.

First, we have to specify the generalisation relation between digraphs.

\section{Definition 6 (Generalisation relation)}

For two digraphs $G_{1}, G_{2}$, we consider that $G_{1}$ is more general than $G_{2}$ iff $G_{1} \rightarrow$ $G_{2}$.

This relation is only a pre-order because the antisymmetry property is not fulfilled.

The same problem occurs in Inductive Logic Programming. To get rid of this problem, Plotkin 14 defined equivalence classes of clauses, and showed that there is a unique representative of each clause, which he named 'the reduced clause'. For this purpose, we define the following equivalence relation between two digraphs.

\section{Definition 7 (AC-equivalence graphs)}

Two digraphs $G_{1}$ and $G_{2}$ are $A C$-equivalent, denoted by $G_{1} \leftrightharpoons G_{2}$, iff both $G_{1}$ $\rightarrow G_{2}$ and $G_{2} \rightarrow G_{1}$.

For example in the Figure 1 we have $G_{1} \rightarrow G_{2}$ but we also have $G_{2} \rightarrow G_{1}$ with the labelling $\mathcal{I}: \mathcal{I}\left(a_{4}\right)=\left\{a_{0}\right\}, \mathcal{I}\left(a_{10}\right)=\left\{a_{0}\right\}, \mathcal{I}\left(b_{5}\right)=\left\{b_{1}\right\}, \mathcal{I}\left(b_{9}\right)=\left\{b_{1}\right\}, \mathcal{I}\left(c_{7}\right)=$ $\left\{c_{3}\right\}, \mathcal{I}\left(c_{8}\right)=\left\{c_{3}\right\}, \mathcal{I}\left(c_{6}\right)=\left\{c_{2}, c_{3}\right\}$.

Using this equivalence relation, we can define equivalence classes of digraph.

\subsection{AC-Projection and Reduction}

We have an equivalence relation between graphs using the AC-projection. In this paragraph we study the properties of this operation and search for a reduced element in an equivalence class of graphs. For this purpose, we define two reduction operators. Using these operators we construct an AC-equivalent digraph by removing (first operator) or merging (second operator) vertices.

\section{Definition 8 (AC-redundant vertex)}

For a digraph $G$, for a vertex $x \in V(G)$, if $G \leftrightharpoons G$ - $x$ then $x$ is an $A C$-redundant vertex. (With $G-x=G^{\prime}$ s.t $V\left(G^{\prime}\right)=V(G)-\{x\}$ and $A\left(G^{\prime}\right)=A(G)-\{(y, z) \mid y=x$ or $z=x\})$.

In the Figure 3 the node 1 labelled "c" is AC-redundant.

\section{Definition 9 (AC-equivalent vertices)}

For a digraph $G$, we say that $x_{1}, x_{2} \in V(G)$ are $A C$-equivalent iff for the $A C$ projection $\mathcal{I}: G \rightarrow G, \mathcal{I}\left(x_{1}\right)=\mathcal{I}\left(x_{2}\right)$.

In the Figure 3 the nodes with same label are, in this case, AC-equivalent.

These two definitions give a reduction operator. 


\section{Procedure: $\mathcal{R}$}

Data: a labelled digraph $\mathrm{G}$

Result: a labelled digraph $\mathrm{G}^{\prime} / /$ with $\mathrm{G}^{\prime} \leftrightharpoons \mathrm{G}$

$\mathrm{G}^{\prime}:=\mathrm{G}$;

next := true;

while next do

next:=false;

if (there is a $A C$-redundant vertex $x \in V\left(G^{\prime}\right)$ ) then

$\mathrm{G}^{\prime}:=\mathrm{G}^{\prime}-\mathrm{x}$

next:=true;

end

if there is a set of $A C$-equivalent vertices $E=\left\{x_{1}, \ldots x_{n}\right\} \in V\left(G^{\prime}\right)$ with $|E|>1$ then

// Merge of the AC-equivalent vertices of $\mathrm{E}$

add a vertice $\mathrm{x}$ to $\mathrm{V}\left(\mathrm{G}^{\prime}\right)$ with $\mathrm{P}(\mathrm{x})=\bigcup \mathrm{P}\left(x_{i}\right)$ and $\mathrm{S}(\mathrm{x})=\bigcup \mathrm{S}\left(x_{i}\right)$;

$/ /$ remove all $x_{i} \in \mathrm{E}$ from $\mathrm{G}^{\prime}$

$\mathrm{G}^{\prime}:=\mathrm{G}^{\prime}-\mathrm{E}$;

next:=true;

end

end

return $G^{\prime}$;

This $\mathcal{R}$ operation is polynomial because the AC-projection is polynomial.

\section{Proposition 3 ( $\mathcal{R}$ equivalence)}

$\mathcal{R}(G) \leftrightharpoons G$

Proof.

1) If we remove an $A C$-redundant vertex $x \in V(G)$, by definition $G \leftrightharpoons G-x$.

2 ) if we merge a set of AC-equivalent vertices $\mathrm{E}=\left\{x_{1}, \ldots x_{n}\right\}$ with $|E|>1$ in a vertex $\mathrm{x}$, we obtain a new graph $\mathrm{G}^{\prime}$. We have to prove that $\mathrm{G} \leftrightharpoons \mathrm{G}^{\prime}$

We have a AC-projection $\mathcal{I}$ from $G$ into $G$

a) $\mathrm{G} \rightarrow \mathrm{G}^{\prime}$

We construct a labeling $\mathcal{I}^{\prime}$ from $\mathrm{G}$ into $\mathrm{G}^{\prime}$ with

for $x_{i} \in \mathrm{E}, \mathcal{I}^{\prime}\left(x_{i}\right)=\mathrm{x}$

for $y_{j} \notin \mathrm{E}$, if $\mathcal{I}\left(y_{j}\right) \bigcap \mathrm{E} \neq \emptyset$ then

$\mathcal{I}^{\prime}\left(y_{j}\right)=\left(\mathcal{I}\left(y_{j}\right)-\mathrm{E}\right) \bigcup \mathrm{x}$

else

$\mathcal{I}^{\prime}\left(y_{j}\right)=\mathcal{I}\left(y_{j}\right)$

We know that $\forall x_{i} \in \mathrm{E}$, and $\forall y_{j} \in \mathrm{S}(\mathrm{x}), \mathcal{I}\left(x_{i}\right) \sim \succ \mathcal{I}\left(y_{j}\right)$. Since $\mathrm{S}(\mathrm{x})=\bigcup \mathrm{S}\left(x_{i}\right) \mid x_{i}$ $\in \mathrm{E}$ we have $\mathcal{I}^{\prime}\left(x_{i}\right)=\{\mathrm{x}\} \sim \succ \mathcal{I}^{\prime}\left(y_{j}\right)$ and reciprocally for the predecessors.

So $\mathcal{I}^{\prime}\left(y_{j}\right)$ is an AC-projection.

b) $\mathrm{G}^{\prime} \rightarrow \mathrm{G}$

We construct a labeling $\mathcal{I}^{\prime \prime}$ from $\mathrm{G}^{\prime}$ into $\mathrm{G}$ with

for $\mathrm{x} \in \mathrm{V}\left(\mathrm{G}^{\prime}\right), \mathcal{I}^{\prime \prime}(x)=\mathrm{E}$

for $\mathrm{y} \in \mathrm{V}\left(\mathrm{G}^{\prime}\right)$ and $\mathrm{y} \neq \mathrm{x}, \mathcal{I}^{\prime \prime}(y)=\mathcal{I}(y)$ 
We know that for all $x_{i} \in \mathrm{E}$ and $y_{j} \in \mathrm{S}\left(x_{i}\right), \mathcal{I}\left(x_{i}\right) \sim \succ \mathcal{I}\left(y_{j}\right)$ (and reciprocally for the predecessors).

Since $\mathcal{I}^{\prime \prime}(x)=\mathcal{I}(x)$ and $\mathcal{I}^{\prime \prime}(y)=\mathcal{I}(y)$, we have $\mathcal{I}^{\prime \prime}\left(x_{i}\right) \sim \succ \mathcal{I}^{\prime \prime}\left(y_{j}\right)$ then $\mathcal{I}^{\prime \prime}$ is an AC-projection.

The Figure 3 shows the application of the $\mathcal{R}$ reduction operator on a digraph.

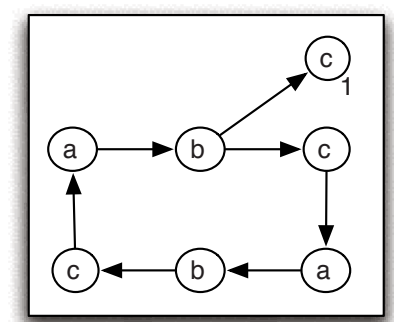

G

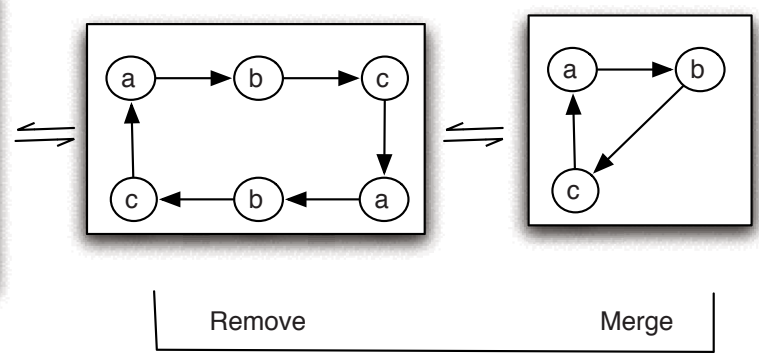

$R(G)$

Fig. 3. $\mathcal{R}(\mathrm{G})$

\subsection{AC-Projection and Generalization Operator}

There are some pairs, (representation languages and generality relations), which have a least general generalization operator . For logic formula and $\theta$-subsumption, this operator is the classical lgg (or rlgg) introduced by plotkin [14. For graph and homomorphism this operator is the graph product [10]. In mathematics this kind of operator is defined as a product operator [15].

Definition 10 (Product operator). A binary operator $\bullet$ is a product operator iff for a pre order (or a partial order) $\geqslant$ between element $E_{i}$

$-E_{1} \bullet E_{2} \geqslant E_{1}$

$-E_{1} \bullet E_{2} \geqslant E_{2}$

- if $E \geqslant E_{1}$ and $E \geqslant E_{2}$ then $E \geqslant E_{1} \bullet E_{2}$

For digraph we have the following product operator $(\otimes)$ for the homomorphism pre-order [16:310].

Definition 11 (Product operator $\otimes$ for digraphs and homomorphism) For two digraphs $G_{1}$ and $G_{2}$ We construct $G=G_{1} \otimes G_{2}$ with

- $L(G)=L\left(G_{1}\right) \bigcap L\left(G_{2}\right)$

- $V(G) \subseteq V\left(G_{1}\right) \times V\left(G_{2}\right)=\left\{x \mid x=\left(x_{1}, x_{2}\right)\right.$ with $\left.l(x)=l\left(x_{1}\right)=l\left(x_{2}\right)\right\}$

- $A(G)=\left\{\left(x, x^{\prime}\right) \mid x=\left(x_{1}, x_{2}\right), x^{\prime}=\left(x_{1}^{\prime}, x_{2}^{\prime}\right)\right.$ and $\left(x_{1}, x_{1}^{\prime}\right) \in V\left(G_{1}\right),\left(x_{2}, x_{2}^{\prime}\right) \in V\left(G_{2}\right)$ \}$\subseteq A\left(G_{1}\right) \times A\left(G_{2}\right)$

For the AC-projection we have also a generalization operator. 


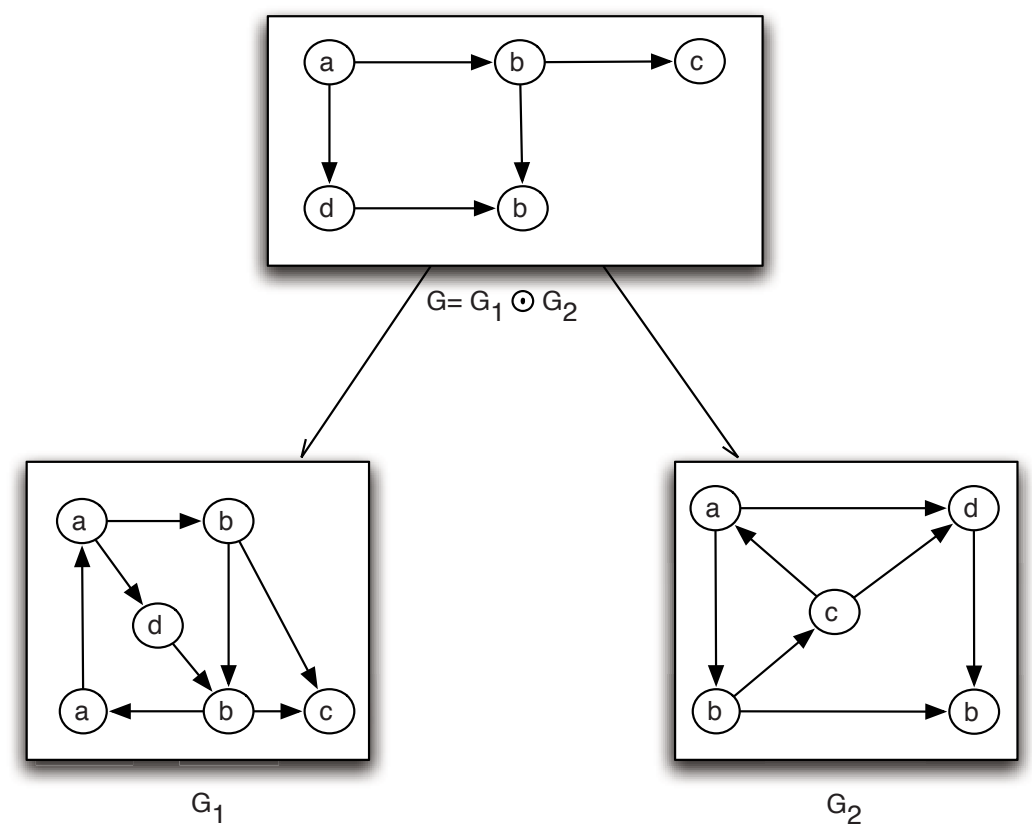

Fig. 4. Product $\odot$ of two digraphs

\section{Proposition 4 (Product operator $\odot$ for digraphs and AC-projection)}

For two digraphs $G_{1}, G_{2}$. The binary operator $G_{1} \odot G_{2}=\mathcal{R}\left(G_{1} \otimes G_{2}\right)$ is a product operator.

So for $G=G_{1} \odot G_{2}$ we have:

1) $G \rightarrow G_{1}, G \rightarrow G_{2}$

2) for a digraph $G^{\prime}$ if $H \rightarrow G_{1}$ and $G^{\prime} \rightarrow G_{2} \Rightarrow G^{\prime} \neg G$

\section{Proof}

1) For two digraphs $G_{1}, G_{2}$, and $\mathrm{G}=G_{1} \otimes G_{2}$ we have $\mathrm{G} \mapsto G_{1}$ and $\mathrm{G} \mapsto G_{2}$ (by property of the digraph product operation [10]). And we know:

- $\mathcal{R}(G) \leftrightharpoons G$ (proposition 5 ).

- if $G \mapsto G_{1} \Rightarrow G \rightarrow G_{1}$ (proposition 1)

2) We know that $\mathrm{G}^{\prime} \rightarrow G_{1}$ and $\mathrm{G}^{\prime} \rightarrow G_{2}$. So there is two labelings $\mathcal{I}_{1}$ and $\mathcal{I}_{2}$ with for each $\mathrm{x} \in \mathrm{V}\left(\mathrm{G}^{\prime}\right), \mathcal{I}_{1}(\mathrm{x})=\left\{x_{1}^{1}, \ldots, x_{1}^{n}\right\} \subseteq \mathrm{V}\left(G_{1}\right)$ and $\mathcal{I}_{2}(\mathrm{x})=\left\{x_{2}^{1}, \ldots, x_{2}^{m}\right\} \subseteq$ $\mathrm{V}\left(G_{2}\right)$.

In $G_{1} \otimes G_{2}$ we have all the couples $\left(x_{1}^{1}, x_{2}^{1}\right),\left(x_{1}^{1}, x_{2}^{2}\right), \ldots\left(x_{1}^{n}, x_{2}^{m}\right)$ by construction. We define the following labelling $\mathcal{I}$ : $\mathrm{G}^{\prime} \rightarrow G_{1} \otimes G_{2}$ with $\mathcal{I}(\mathrm{x})=\mathcal{I}_{1}(\mathrm{x}) \times \mathcal{I}_{2}(\mathrm{x})$. If $(\mathrm{x}, \mathrm{y}) \in \mathrm{V}\left(\mathrm{G}^{\prime}\right)$ we have to prove that $\mathcal{I}(\mathrm{x}) \sim \succ \mathcal{I}(\mathrm{x})$.

For each $\left(x_{1}^{i}, x_{2}^{j}\right) \in \mathcal{I}(\mathrm{x})$ there is $\left(y_{1}^{a}, y_{2}^{b}\right) \in \mathcal{I}(\mathrm{x})$ with $\left(x_{1}^{i}, y_{1}^{a}\right) \in \mathrm{V}\left(G_{1}\right)$ and $\left(x_{2}^{j}, y_{2}^{b}\right) \in \mathrm{V}\left(G_{2}\right)$. Because $x_{1}^{i} \in \mathcal{I}_{1}(\mathrm{x})$ there is, at least, one $y_{1}^{a} \in \mathcal{I}_{1}(\mathrm{y})$ with $\left(x_{1}^{i}, y_{1}^{a}\right) \in \mathrm{V}\left(G_{1}\right)$. Since $x_{2}^{j} \in \mathcal{I}_{2}(\mathrm{x})$ there is, at least, one $y_{2}^{b} \in \mathcal{I}_{2}(\mathrm{y})$ with $\left(x_{2}^{j}, y_{2}^{b}\right)$ $\in \mathrm{V}\left(G_{2}\right)$. Since, the graph product $G_{1} \otimes G_{2}$ builds all the couple $\left(x_{1}^{i}, x_{2}^{j}\right)$ and 
$\left(y_{1}^{a}, y_{2}^{b}\right)$ (with same labels). $\left(\left(x_{1}^{i}, x_{2}^{j}\right),\left(y_{1}^{a}, y_{2}^{b}\right)\right) \in \mathrm{V}(\mathrm{G})$ iff $\left(x_{1}^{i}, y_{1}^{a}\right) \in \mathrm{V}\left(G_{1}\right)$ and $\left(x_{2}^{j}, y_{2}^{b}\right) \in \mathrm{V}\left(G_{2}\right)$. So for $\left(x_{1}^{i}, x_{2}^{j}\right) \in \mathcal{I}(\mathrm{x})$ there is, by definition of $\mathcal{I},\left(y_{1}^{a}, y_{2}^{b}\right) \in$ $\mathcal{I}(\mathrm{y})$ then $\mathcal{I}(\mathrm{x}) \sim \succ \mathcal{I}(\mathrm{y})$. $\mathcal{I}$ is an AC-projection from $\mathrm{G}^{\prime} \rightarrow G_{1} \otimes G_{2}$. Since $\mathcal{R}(\mathrm{G})$ $\leftrightharpoons \mathrm{G}$ (by construction) then $\odot$ is a product operator.

In Figure $4 \mathrm{G}$ is the product of $G_{1}$ and $G_{2}$. It represents all the different 11 subtrees common at $G_{1}$ and $G_{2}$.

\section{Concept Lattice and AC-Projection}

We have a generalization operator and a pre-order between digraphs. With this knowledge, we can define the notion of concept [13].

Definition 12 (concept, $\vee, \geq$ )

For a set of examples $\mathcal{E}$, each example $e \in \mathcal{E}$ is described by a digraph $d(e) \in D$ (description space).

For a digraph $G$, we note $\alpha(G)=\left\{e_{i} \in \mathcal{E} \mid G \rightarrow d\left(e_{i}\right)\right\}$.

For $E_{1} \subseteq \mathcal{E}$, we note $\beta\left(E_{1}\right)=\odot_{e \in H} d()$.

$A$ concept is a couple $\left(E_{1}, G_{1}\right)$ with $E_{1} \subseteq E, G_{1}$ a digraph with $\alpha\left(G_{1}\right)=E_{1}$ and $\beta\left(E_{1}\right) \leftrightharpoons G_{1}$.

For two concepts $\left(E_{1}, G_{1}\right),\left(E_{2}, G_{2}\right)$ :

$\left(E_{1}, G_{1}\right) \vee\left(E_{2}, G_{2}\right)=\left(\alpha(G), G=G_{1} \odot G_{2}\right)\left(E_{1}, G_{1}\right) \geq\left(E_{2}, G_{2}\right)$ iff $G_{1} \rightarrow G_{2}$

\section{Proposition 5 (AC concept lattice)}

For a set of examples $\mathcal{E}$, each example $e \in \mathcal{E}$ is described by a digraph $d(e) \in D$. The correspondance $\alpha, \beta$ defines a Galois connection between $2^{\mathcal{E}}$ and $D$.

Proof. see [10]

This proposition gives the structure of the search space (a concept semilattice). The size of the concept lattice is limited by the minimum of SD and SP where $\mathrm{SD}$ is the size of the description space and SP the size of the partition space. The size SD is very large for relational description but SP is limited by $2^{n}$ where $\mathrm{n}$ is the number of examples.

If we use our method on the set of examples of [10] we obtain the following joinsemilattice Figure 5. In this concept semilattice, each node represent a concept with an extension part: a subset of the set of examples and an intension part :a digraph. The partial order between the elements of the lattice is based on AC-projection, then the digraph, intension part of a concept, can be interpreted as a compact description of a very large (potentially infinite) set of trees. But, thanks to AC-projection, we don't have to explore all the elements of this set as in classical tree mining method [8]. Using this example, we obtain a lattice which is isomorphic with the one given by Graal [10] but it is not always the case. This comes from the fact that, for this set of graphs, the set of included paths is enough to obtain this lattice.

\footnotetext{
${ }^{1}$ For the homomorphism relation.
} 


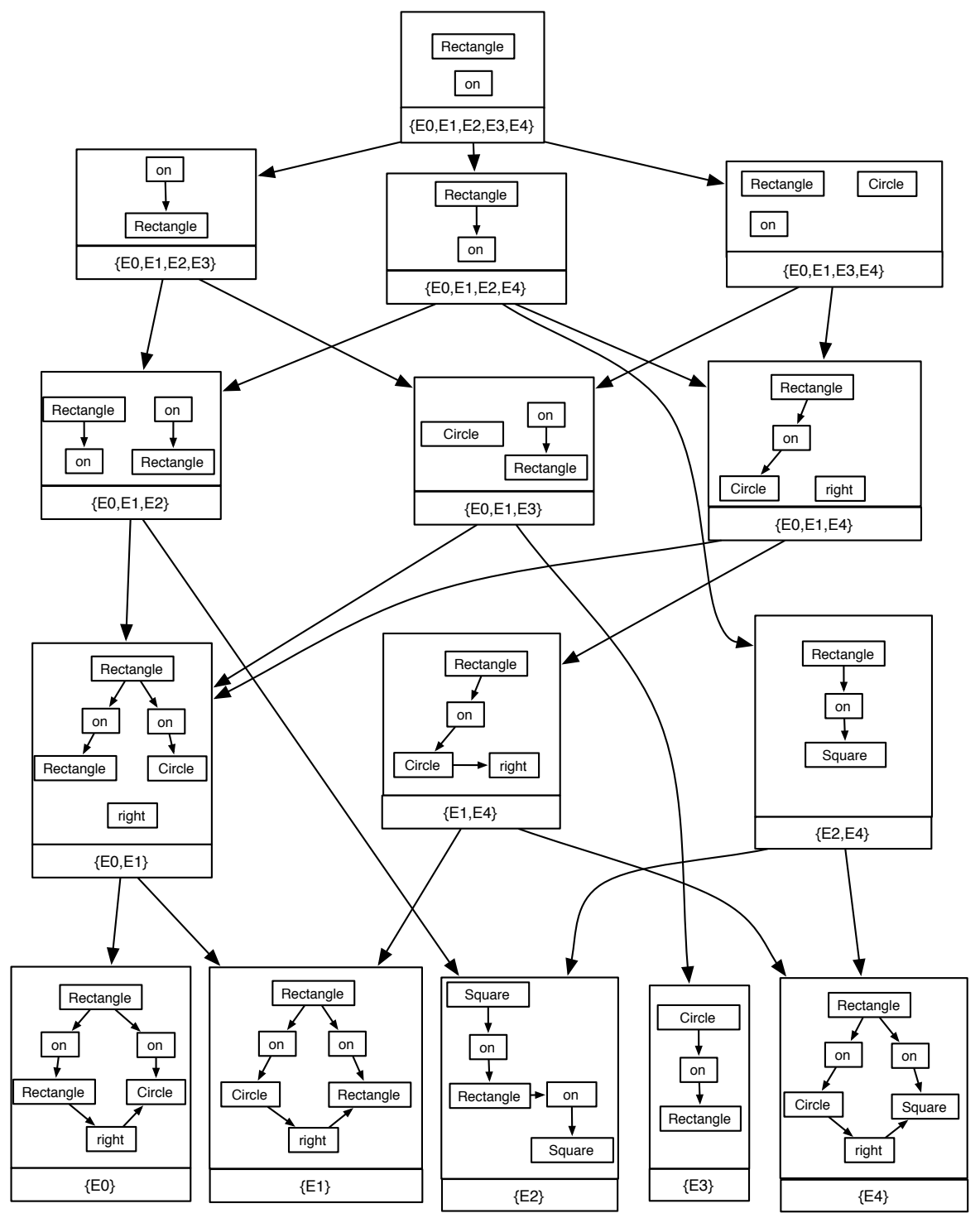

Fig. 5. A set of examples and the concept semilattice for AC-projection

\section{Conclusion}

This study has attempted to merge ideas from different communities: Graph, ILP, CSP. In these communities, the same problem is examined from different points of view: graph and homomorphism, logic and $\theta$-subsumption, and constraint satisfaction problems and resolution. 
Merging these knowledges we have obtained:

- the definition of a new generality relation between graphs with polynomial complexity.

- the definition of a least general generalization operator.

These results can be used for the construction of a concept lattice where the intention part of a concept is described by a digraph. The advantages of this approach compare with the approach [10] are:

- We use general digraph for the description of the examples. In the paper [10] we have a polynomial complexity only for a specific class of graphs.

- All the operations are polynomial (equivalence, reduction, product and concept order).

- We find graphs which express a large set of trees in a compact form.

- The size of the concept lattice is smaller.

But, since the AC projection is less precise, the classifications obtained are also less precise. However, we have a generalization operator, between two digraphs, which gives a digraph which represents all the homomorphic trees belonging at this two digraphs. So, a large part of their common structure is caught.

Acknowledgements. The author is grateful to C. Bessiere and F. Koriche for their constructive remarks.

\section{References}

1. Sowa, J.F.: Conceptual structures: information processing in mind and machine. Addison-Wesley Longman Publishing Co. Inc. Boston, MA, USA (1984)

2. Baget, J.F., Mugnier, M.L.: Extension of simple conceptual graphs: the complexity of rules and constraints. Journal of Artificial Intelligence Research 16, 425-465 (2002)

3. Hell, P., Nesetril, J.: Graphs and homomorphism. In: Oxford Lecture Series in Mathematics and its Application, vol. 28, Oxford University Press, Oxford (2004)

4. Mugnier, M., Chein, M.: Polynomial algorithms for projection and matching. In: ICCS. LNCS (LNAI), vol. 754, pp. 239-251. Springer, Heidelberg (1992)

5. Croitoru, M., Compatangelo, E.: A combinatorial approach to conceptual graph projection checking. In: 24th Int'l Conf. of the British Computer Society's Specialist Group on Art'l Intell, pp. 239-251. Springer, Heidelberg (2004)

6. Pfeiffer, H.: A comparison of different conceptual structures projection algorithms, Submitted to ICCS'07 (2007)

7. Maloberti, J., Sebag, M.: Fast theta-subsumption with constraint satisfaction algorithms. Machine Learning 55, 137-174 (2004)

8. Zaki, M.: Efficiently mining frequent trees in a forest. In: 8th Intl. Conf. knowledge discovery and data mining, pp. 71-80 (2002)

9. Cook, D.J., Holder, L.B.: Substructure discovery using minimum description length and background knowledge. Journal of Artificial Intelligence Research 1, 231-255 (1994) 
10. Liquiere, M., Sallantin, J.: Structural machine learning with galois lattice and graphs. In: Shavlik, J.W. (ed.) ICML, pp. 305-313. Morgan Kaufmann, San Francisco (1998)

11. Sebag, M., Rouveirol, C.: Resource-bounded relational reasoning: induction and deduction through stochastic matching. Machine Learning 38, 41-62 (2000)

12. Bessiere, C.: Constraint propagation. In: Rossi, F., van Beek, P., Walsh, T. (eds.) Handbook of Constraint Programming, Elsevier, Amsterdam (2006)

13. Ganter, B., Wille, R.: Formal Concept Analysis: Mathematical Foundations. Springer-Verlag, New York, Inc. Secaucus, NJ, USA, Translator-C. Franzke (1997)

14. Plotkin, G.: A note on inductive generalization. Machine Intelligence 5, 153-163 (1970)

15. Crole, R.: Categories for Types. Cambridge Mathematical Textbooks. Cambridge University Press, Cambridge (1993)

16. Weichsel, P.M.: The kronecker product of graphs. Proc.Am.Math.Soc. 13, 47-52 (1962) 